\title{
Confianza en instituciones políticas: factores que explican la percepción de confianza en Chile
}

\author{
Víctor Saldaña Zúñiga ${ }^{1}$ \\ Manuel Pineda Torres ${ }^{2}$
}

Fecha de recepción: 24 de agosto de 2019

Fecha de aprobación: 17 de octubre de 2019

Fecha de publicación: 31 de diciembre de 2019

\begin{abstract}
Resumen
El presente artículo contribuye en la exploración de los factores que inciden en la confianza de la población en las instituciones políticas. La problemática asociada a instituciones políticas en contextos democráticos ha adquirido relevancia tanto a nivel nacional como internacional. En el caso chileno, se destaca el aumento progresivo de la desconfianza en las instituciones políticas, particularmente hacia los partidos, acompañado de un clima político de desafección y de baja participación política. Con el objetivo de explicar este fenómeno, se presenta un modelo de regresión lineal múltiple basado en tres indicadores ampliamente respaldados por la literatura sobre confianza política: percepción de eficacia, bienestar económico y percepción de corrupción, constituidos a partir de la base de datos Latinobarómetro 2017. Los resultados obtenidos ponen en evidencia la relevancia de estos factores asociados evaluación subjetiva del desempeño institucional para explicar la confianza política en Chile. No obstante, cada uno de estos factores posee una particularidad explicativa con respecto al caso estudiado.

Palabras claves: Confianza política, desafección, eficacia institucional, percepción de bienestar económico, percepción de corrupción.
\end{abstract}

\section{Trust in political institutions: factors to explain the perception of confidence in Chile}

\begin{abstract}
This article contributes to the exploration of the factors that influence the people's trust in political institutions. The problems associated with politi-
\end{abstract}

1 Magíster en Ciencias Sociales, mención sociología de la modernización, Universidad de Chile, Santiago de Chile. Universidad de Chile, Santiago de Chile, Chile. Contacto: victor.saldana@uchile.cl

2 Magíster en Ciencias Sociales, mención sociología de la modernización, Universidad de Chile. Académico de las Escuelas de Trabajo Social y Sociología de la Universidad Católica Silva Henríquez-Universidad San Sebastián. Santiago de Chile. Contacto: mpinedat@ucsh.cl 
cal institutions in democratic contexts have gained relevance both nationally and internationally. In the case of Chile, the progressive higher distrust in political institutions, particularly towards the political parties, is accompanied by a political climate of disaffection and low political participation. With the aim of explaining this phenomenon, a multiple linear regression model is presented based on three indicators widely supported by the literature on political trust: perception of efficacy, economic well-being and perception of corruption, constituted from the Latinbarometer 2017 database. The results obtained highlight the relevance of these associated factors, subjective evaluation of institutional performance to explain political confidence in Chile. However, each of these factors has an explanatory particularity with respect to the case under review.

Key words: disaffection, institutional effectiveness, trust in politics, perception of economic well-being, perception of corruption.

\title{
Confiança nas instituições políticas: fatores que explicam a percepção de confiança no Chile
}

\begin{abstract}
Resumo
O presente artigo contribui para a exploração dos fatores que incidem na confiança da população nas instituições políticas. A problemática associada a instituições políticas em contextos democráticos adquiriu relevância tanto em nível nacional como internacional. No caso chileno, destaca-se o aumento progressivo da desconfiança nas instituições políticas, particularmente dos partidos, acompanhado de um clima político de desafeto e de baixa participação política. Com o objetivo de explicar este fenômeno, é apresentado um modelo de regressão linear múltiplo baseado em três indicadores amplamente respaldados pela literatura sobre confiança política: percepção de eficiência, bem-estar econômico e percepção de corrupção, constituídos a partir da base de dados Latinobarómetro 2017. Os resultados obtidos evidenciam a relevância destes fatores associados à avaliação subjetiva do desempenho institucional para explicar a confiança política no Chile. Porém, cada um destes fatores possui uma particularidade explicativa com relação ao caso estudado.
\end{abstract}

Palavras-chave: Confiança política, desafeto, eficiência institucional, percepção de bem-estar econômico, percepção de corrupção.

\section{Introducción}

La preocupación por la calidad de las democracias liberales representativas, sean estas de larga data o recientes, es una agenda de investigación que aún sigue abierta. Una de las principales razones de esta preocupación surge a raíz de la mantenida desafección y la baja participación de los ciudadanos en las instancias 
formales de participación política, como los procesos electorales o la adscripción a un partido político. Entre las dimensiones de este problema destaca la poca confianza en las instituciones políticas por parte de la ciudadanía, particularmente, hacia los partidos políticos (Torcal, 2014; del Tronco, 2012).

El diagnóstico y pronóstico sobre este síntoma de las democracias actuales sigue abierto. Mientras que unos señalan que estos bajos niveles de confianza se deben más a un desánimo de los ciudadanos con sus representantes y su accionar en vez de una afrenta en contra la democracia, la cual podría seguir sosteniéndose pese a la baja (Hardin, 2010; Runciman, 2019), otros consideran que esto se debería a los efectos del neoliberalismo sobre el sistema político — de lo que denominan como posdemocracias, en las que los intereses de las élites económicas se verían mayormente representados que las de los propios ciudadanos, provocando su distanciamiento (Crouch, 2004; Jörke, 2008; Offe, 2014). A estos diagnósticos se suma la evidencia que la crisis económica, las políticas de austeridad y la incertidumbre generada por las mismas en la población ha profundizado aún más la crisis de confianza en las instituciones y representantes, propiciando el surgimiento líderes y partidos de carácter populista (Cossarini \& Vallespín, 2019; Norris \& Inglehart, 2019; Simón, 2019).

Ante esta dinámica global de la confianza política, el caso de Chile no es la excepción. Pese a ser una de las democracias más estables de Latinoamérica, y cumpliendo en gran medida con los indicadores democráticos internacionales - Democracy Index y Freedom House-, los niveles de confianza política siguen siendo bajos, decreciendo de manera sostenida en relación con los indicadores de satisfacción con el régimen. Por este motivo, el Democracy Index (Economist Intelligence Unit, 2018) clasifica a Chile en el segundo lugar de la región - luego de Uruguay—, pero como una "democracia defectuosa o imperfecta", debido a los bajos niveles de participación y a una cultura política poco desarrollada. 
Las encuestas nacionales de los últimos años ratifican la baja confianza en las instituciones políticas, particularmente en los partidos políticos (Centro de Estudios de Conflicto y Cohesión Social, 2015; Centro de Estudios Públicos, 2017). Esto, en medio de la aparición en los últimos años de escándalos de corrupción tanto políticos como económicos, así como de diferentes movimientos sociales que han protagonizado protestas masivas, como los casos del movimiento estudiantil por la educación pública o el movimiento en contra del sistema privado de administración de fondo de pensiones (No + AFP), que han contribuido a la profundización de la desconfianza y a un ambiente de crisis política (Siavelis, 2016). Esto ha llevado a discusiones respecto a si en Chile existe una crisis de representación o de legitimidad institucional (Aninat \& González, 2016; Joignant, Fuentes \& Morales, 2017).

A partir del caso de Chile, el objetivo de este artículo es explicar los niveles de confianza en las instituciones políticas a partir de los datos de la encuesta del Latinobarómetro 2017, poniendo a prueba la capacidad predictiva de tres índices construidos en base antecedentes teóricos relevantes: evaluación de la eficacia, percepción de bienestar económico y percepción de corrupción pública. Para cumplir con este objetivo, este artículo se compone de cinco apartados, considerando esta introducción. En el segundo apartado se abordan los antecedentes teóricos de la discusión sobre confianza política, con énfasis en la literatura sobre el impacto de las tres variables antes mencionadas sobre la confianza en las instituciones. En el tercer apartado, se exponen los aspectos metodológicos, describiendo la construcción de los índices y la validez de los mismos, y el modo de análisis estadístico elegido. En el cuarto apartado, se exponen los resultados arrojados por el modelo de regresión lineal múltiple. Finalmente, en el quinto apartado se exponen las conclusiones de este estudio, discutiendo sus aportes y limitaciones para el estudio de la confianza política en Chile, así como también algunas sugerencias para futuros estudios. 


\section{Antecedentes teóricos}

La discusión sobre la confianza en las instituciones políticas tiene larga data en los avances teóricos, particularmente en relación con el desarrollo de los sistemas políticos modernos y la legitimidad y estabilidad de los mismos en los regímenes democráticos (Almond \& Verba, 1989; Easton, 1975; Lipset, 1977). Como indican del Tronco (2012) y Segovia (2006), la confianza en las instituciones es elemental para dar luces sobre los niveles de legitimidad que tienen los gobiernos democráticos. Para Lechner (2016), es un sentimiento público en que se gesta la creencia en la legitimidad, que da cuenta del lazo entre los ciudadanos y sus instituciones centrales en la que los ciudadanos tienen la creencia de que sus instituciones actuarán y que obtendrán resultados según lo que se espera o desea de estas (Przeworski, Álvarez, Cheibub \& Limongi, 1996), sin necesidad de ejercer un control o supervisión permanente sobre las mismas (Segovia, 2006). Ello implica criterios como que las instituciones actuaran de manera correcta y sin perjudicar los intereses propios o de la nación (Montero, Zmerli \& Newton, 2008). Por estas razones, la confianza política representa uno de los principales indicadores del sentimiento básico y subjetivo que deberían tener los ciudadanos con sus instituciones y el orden político que representan según sus expectativas (Lechner, 2016; Newton \& Norris 2000; Megías, 2019).

Dentro de la literatura de confianza política predominan dos enfoques centrales (Morales, 2008; del Tronco 2012): el racional-institucional y el enfoque culturalista. El primer enfoque considera la confianza política como la satisfacción de los individuos en relación a las instituciones en un contexto determinado, en función de que estas garanticen una serie de derechos y libertades civiles; un entorno estable y próspero tanto política como económicamente; y de que estas actúan acorde a estándares legales que aseguran su accionar ético y transparente (Zmerli \& Castillo, 2015; Levi \& Stoker, 2000; Marien, 2011; Mishler \& Rose, 2001). 
El segundo enfoque sostiene que la confianza política es explicada por factores vinculados a la aprehensión de normas y valores por medio de procesos de socialización, participación en asociaciones voluntarias y confianza interpersonal (exo-endogrupo), también conocida como confianza social. La tríada se vincula con la confianza interpersonal, y la confianza política ya sería un mismo tipo de creencia (Putnam, 2001; Segovia, 2006). La confianza interpersonal se da a temprana edad por medio de la socialización y más tarde se proyecta en agentes secundarios de socialización, en la interacción con pares y participación en organizaciones sociales o políticas de carácter voluntario (Putnam, 2011). Lo último produciría un círculo virtuoso donde los ciudadanos se interesarían más por los asuntos públicos, formando mayores lazos de reciprocidad al interior de la comunidad política, impactando en la prosperidad social, en la confianza hacia las instituciones y en el fortalecimiento de la democracia (Durand, 2006; Stoyan, Niedzwiecki, Morgan, Hartlyn \& Espinal, 2016). Sin embargo, el enfoque presenta algunas aprensiones. Por ejemplo, Hardin (2010) indica que, en términos conceptuales y epistemológicos, la confianza interpersonal ordinaria no puede ser igualada a la confianza política, ya que esta última no tiene como base la relación de un individuo con otro depositario de la confianza, sino de un individuo con una organización o institución.

Desde el enfoque racional-institucional, también llamado instrumental, la eficiencia y la eficacia de las instituciones y gobiernos (Levi \& Stoker, 2003) son indicadores centrales de los niveles de confianza, particularmente en la evaluación que hace la población de la situación económica. Esta última suele ser considerada su principal dimensión, ya que supone que un ambiente estable económicamente se debe al desempeño de las autoridades y representantes políticos, lo que promueve una mayor confianza en estos (Mishler \& Rose, 2005; Morales, 2008; Torcal, 2014). Miller y Listhaug (1990) consideran que es una de las variables más relevantes para evaluar el apoyo a los gobiernos e instituciones. 
Además del impacto del desempeño institucional, para este enfoque, un buen desarrollo, estabilidad y desempeño macroeconómico de los gobiernos impacta positivamente en la confianza política (Listhaug \& Ringdal, 2008). Es a partir de esta que la población realiza una evaluación en base a su percepción subjetiva de la situación económica a nivel general y particular (Norris \& Inglehart, 2018; van der Meer, 2018; van der Meer \& Deeker, 2011). Megías (2019), al estudiar la desafección en España, señala que la gestión por parte de las autoridades de la crisis económica de 2008 y sus efectos, exacerbó la desconfianza y el sentimiento de que los políticos son poco capaces y que velan por sus intereses.

En las investigaciones donde se utilizan indicadores económicos objetivos, los resultados han sido variados. Por ejemplo, Hakhverdian y Mayne (2012), al realizar un análisis multinivel en diferentes naciones, concluyen que los indicadores de performance económica nacional no son relevantes para explicar las variaciones en la confianza política. Así, Vilhelmsdóttir y Helgi (2018), al estudiar los efectos de la crisis financiera de 2008, descubren que la relación positiva entre confianza política y factores macroeconómicos no se daría en todos los casos y que en ella podrían mediar otras condiciones contextuales, mientras que Kroknes, Jakobsen y Grønning (2015) consideran que la crisis financiera tuvo una influencia negativa en la confianza política en los países en donde la crisis afectó fuertemente a la economía nacional. Pese a lo anterior, diversos estudios (Elinas \& Lamprianou, 2014; van der Meer \& Hakhverdian, 2017; Zmerli \& Castillo, 2015) han comprobado que las evaluaciones subjetivas del desempeño económico nacional se correlacionan fuertemente con la confianza política a través de diferentes países, inclusive en países cuyo régimen son semidemocráticos o no-democráticos (véase Park, 2017), confirmando la perspectiva de que la confianza política varía de acuerdo a la eficacia con que los gobiernos proveen prosperidad y paz (Norris \& Inglehart, 2018).

Otro de los factores que entra en juego al momento de medir la confianza es la percepción de los actos de corrupción política. La 
corrupción usualmente es entendida como la utilización inadecuada del poder público por parte de instituciones y autoridades políticas para conseguir un beneficio privado (Rose-Ackerman, 1999). Así, es ampliamente aceptado que esta práctica tiene un efecto negativo para los regímenes democráticos, la legitimidad de los gobiernos y el desarrollo económico de las naciones (Anderson \& Tverdova, 2003).

Hardin (2010) considera que la corrupción afecta los niveles de confianza política, ya que pone en cuestión la transparencia con la que actúan los gobiernos, lo cual genera una imagen en la que las autoridades no sirven a la mayoría, sino que a grupos con un mayor poder económico. En este sentido, la corrupción dañaría la confianza en aspectos relevantes del funcionamiento de los sistemas políticos, tales como la efectividad y equidad con la que actúan sus instituciones (Miller \& Listhaug, 1990). Esto lleva a que la población cuestione la posibilidad real de que sus demandas e intereses sean atendidos (Della Porta, 2000) y que las promesas hechas por sus autoridades para enfrentar la corrupción sean efectivas (Morris \& Klesner, 2010).

Tal como indican Morris y Klesner (2010), la mayoría de los estudios que vinculan la percepción de corrupción y los niveles de confianza, dejan entrever la relación endógena que existe entre ambos factores: por un lado, una baja confianza por parte de la población produciría un grado de tolerancia mayor hacia la corrupción y mayores expectativas sobre la misma; por el otro, la corrupción disminuiría los niveles de confianza de la población, y con ello, la legitimidad de los regímenes (p. 1259).

Si bien esta mutua causalidad es aún es tema de discusión (Dalton, 2004; Morris \& Klesner, 2010; Wroe, Allen \& Birch, 2012), la tendencia principal es asociar la corrupción como uno de los factores que explica la confianza hacia las instituciones políticas (van der Meer \& Hakhverdian, 2017), asociada al desempeño o performance institucional. Mishler y Rose (2001) comprueban que las naciones con mayores niveles de corrupción presentaban también bajos ni- 
veles de confianza política. Para Chang y Chu (2006), y Anderson y Tverdova (2003) los casos o escándalos de corrupción influyen en la predisposición y actitud negativa de los públicos hacia las autoridades políticas y sus instituciones. Esto se complementa con otros estudios (Simón, 2019; Jiménez, 2013, 2016) que comprueban el efecto que tienen los casos de corrupción sobre la percepción de los ciudadanos de sus organizaciones políticas y sus representantes, sobre todo luego de la visibilización y proliferación de estos casos después las crisis económicas y las medidas de austeridad.

Específicamente, van der Meer (2010) comprueba la relevancia de la corrupción para explicar las variaciones entre diferentes países en los niveles de confianza en el parlamento. En otro estudio, van der Meer y Hakhverdian (2017), a través de un estudio transnacional, concluyen que a medida que la corrupción está más extendida, es menor la confianza hacia las instituciones políticas y mayor es la insatisfacción con el funcionamiento de la democracia. Hakhverdian y Mayne (2012) van más allá, indicando que la corrupción tiene un mayor efecto negativo en sectores de la población donde el nivel educacional es mayor.

\section{Metodología de trabajo y análisis}

\section{Datos a utilizar}

Para la aplicación del modelo, se considerarán variables provenientes de la base de datos Latinobarómetro 2017. La base de datos recopila información de 18 países de Latinoamérica, considerando una muestra total de 20.200 personas, no obstante, en relación con los fines analíticos de este artículo, se seleccionaron solo aquellos casos correspondientes a Chile. El diseño muestral de la encuesta realizada por Latinobarómetro varía acorde a cada país. En el caso del muestreo utilizado en Chile, se puede señalar que fue un muestreo probabilístico trietápico, que consideró un error muestral de +/-2.8, debido a lo cual se contemplaron 1.200 casos. 
Consideraciones procedimentales de fiabilidad para la elaboración de índices

A partir de los antecedentes presentados en la problematización sobre la confianza en las instituciones políticas, se considerarán 3 variables independientes y 1 dependiente. Cada una de estas variables son índices sumativos construidos a partir de diferentes indicadores de tipo Likert. Los indicadores considerados por cada índice poseen las mismas categorías de respuesta. Para asegurar la fiabilidad de estos y la medición efectiva de un concepto latente por cada índice construido, se realizaron pruebas de alfa de Cronbach y Análisis Factorial de componentes principales. Ambas pruebas permitieron sustentar la construcción de indicadores trabajados en el modelo de regresión múltiple.

\section{Pruebas que sustentan la elaboración de índices}

En relación al análisis factorial de componentes principales, este fue empleado para asegurarse que los reactivos de un índice determinado midieran efectivamente un constructo trabajado en los antecedentes teóricos de este artículo. Las consideraciones procedimentales consideradas fueron: 1) Visualizar el cumplimiento de los supuestos asociados a la prueba KMO y la prueba de esfericidad de Bartlett por cada índice construido; 2) considerar la conformación de un solo componente, el cual tuviera una varianza explicada superior al $60 \%$ y que la prueba diera como componente de autovalor una cifra de 1 y/o superior; 3) realización de rotación de tipo Varimax de indicadores, considerando una rotación de carácter ortogonal (López \& Gutiérrez, 2019).

El detalle de cada uno de los índices construidos, sus valores de varianza de explicación y autovalor son los siguientes: 
Tabla 1

Varianza explicada por componentes principales de cada índice

\begin{tabular}{|l|c|c|c|}
\hline Índices & $\begin{array}{c}\text { Cantidad } \\
\text { componentes }\end{array}$ & Autovalor & $\begin{array}{c}\text { Varianza } \\
\text { explicada }\end{array}$ \\
\hline Confianza política & 1 & 2.8 & $70.550 \%$ \\
\hline Eficacia & 1 & 1.6 & $82.953 \%$ \\
\hline $\begin{array}{l}\text { Percepción de bienestar } \\
\text { económico }\end{array}$ & 1 & 2 & $69.847 \%$ \\
\hline Percepción de corrupción & 1 & 3.2 & $81.176 \%$ \\
\hline
\end{tabular}

Nota: Elaboración propia en base a datos de la encuesta Latinobarómetro 2017.

En cuanto a las prueba $\mathrm{KMO}$, todos los tests que consideraron las variables de origen de los índices utilizados en este artículo, obtuvieron un valor de adecuación superior al 0.5. Ello asegura que las correlaciones parciales entre las variables son lo suficientemente pequeñas. Otra condición que cumplen los índices utilizados en el modelo de regresión se relaciona con las significaciones de la esfericidad de Bartlett, en este caso, en las 4 pruebas realizadas, las cuales se corresponden con los 4 índices construidos, el valor de tal prueba fue de 0.000 (López y Gutiérrez, 2019).

Por último, considerando la confiabilidad de las variables consideradas para formar parte de un índice determinado, al realizar la prueba, se tomó en cuenta la cantidad de reactivos que conformarían un índice determinado (que se detalla en el siguiente acápite), los valores fueron los siguientes:

Tabla 2

Nivel de confiabilidad de indices

\begin{tabular}{|l|c|c|}
\hline Índices & $\begin{array}{c}\text { Alfa de } \\
\text { Cronbach }\end{array}$ & $\begin{array}{c}\text { Número de elementos } \\
\text { (variables) }\end{array}$ \\
\hline Confianza Política & 0.85 & 4 \\
\hline Eficacia & 0.79 & 2 \\
\hline Percepción de bienestar económico & 0.78 & 4 \\
\hline Percepción de corrupción & 0.92 & 4 \\
\hline
\end{tabular}

Nota: Elaboración propia en base a datos de la encuesta Latinobarómetro 2017. 
Lo anterior sustenta y respalda que los índices construidos son fiables y que, efectivamente, miden lo que se ha detallado en términos teóricos en este artículo. Claramente, los resultados del análisis factorial de componentes principales y las pruebas de alpha de Cronbach, la cual entregó valores superiores a 0.6 en cada índice (Corbetta, 2007), sustentan la construcción de los índices considerados en el modelo de regresión lineal múltiple.

\section{Detalle de índices: composición e interpretación}

\section{Variable dependiente}

Para el modelo de regresión lineal múltiple, la variable dependiente será un índice de confianza hacia las instituciones políticas (Confianza política). Este índice está compuesto por 3 variables ordinales que consultan por la confianza en las siguientes instituciones: Congreso, gobierno, Poder Judicial y partidos políticos. Las respuestas van del valor 1 (Mucha) al valor 4 (Ninguna). El índice va del valor 4 (Mucha confianza) a 16 (Ninguna confianza). Su media de respuestas es de 13.

\section{Variables independientes}

La primera de ellas es un índice de "Eficacia" compuesto por dos variables de tipo ordinal. Las variables consideradas son: 1) Grado de satisfacción con el funcionamiento de la democracia y 2) funcionamiento de la economía (en general). Sus respuestas van del valor 1 (Muy satisfecho) al valor 4 (Nada satisfecho). El índice va del valor 2 (Muy satisfecho) a 8 (Nada satisfecho). Su media de respuestas es de 5.7. La hipótesis de esta variable es que existe una relación entre el grado de eficacia con el gobierno en ambas áreas y los niveles de confianza hacia las instituciones políticas, mientras que la hipótesis nula (H0) es que no existe relación entre estas variables. 
La segunda es un índice de "Percepción de bienestar económico" y está compuesta por tres variables de tipo ordinal, que toman valores de 1 a 5 . Los indicadores surgen a partir de la siguiente pregunta: Situación económica pasada del país, Situación económica futura del país y Situación económica personal actual. Para los tres indicadores, las respuestas van desde el valor 1 (Mucho mejor) y valor 5 (Mucho peor). En tanto, el índice consigna valores desde el valor 3 (Mucho mejor) a 15 (Mucho peor). Su media de respuestas es de 8.6. La hipótesis de esta variable es que existe relación entre percepción de bienestar económico y la confianza hacia las instituciones políticas, mientras que la hipótesis nula (H0) es que no existe relación entre estas variables.

La tercera variable es un índice de "Percepción de corrupción" que está compuesto por cuatro variables de tipo ordinal, que toman valores de 0 a 10. Los cuatro indicadores consignados son: Grado de corrupción en los Tribunales de Justicia, Grado de corrupción en el gobierno nacional, Grado de corrupción en las municipalidades y Grado de corrupción en el Congreso Nacional. El índice va del valor 0 (Nada de corrupción) al valor 40 (Mucha corrupción). Su media de respuestas es de 25. La hipótesis de esta variable es que existe relación entre percepción de corrupción en instituciones políticas y los niveles de confianza hacia las instituciones políticas. Su hipótesis nula (H0) es que no existe relación entre estas variables.

La tabla 3 presenta los respectivos estadísticos, ya descritos anteriormente, de cada una de las variables a considerar para el modelo de regresión lineal múltiple. De manera posterior, se entrega una breve lectura de los estadísticos presentados. 
Tabla 3

Estadísticos descriptivos

\begin{tabular}{|l|c|c|c|c|c|c|}
\hline & Media & Mediana & Desviación & Min & Max & $\begin{array}{c}\text { Resp. } \\
\text { válidas }\end{array}$ \\
\hline $\begin{array}{l}\text { Confianza } \\
\text { política }\end{array}$ & 13 & 13 & 2.6 & 4 & 16 & 1,16 \\
\hline Eficacia & 5.7 & 6 & 1.3 & 2 & 8 & 1,14 \\
\hline $\begin{array}{l}\text { Percepción } \\
\text { de bienestar } \\
\text { económico }\end{array}$ & 8.6 & 9 & 2 & 3 & 15 & 1,11 \\
\hline $\begin{array}{l}\text { Percepción } \\
\text { de corrup- } \\
\text { ción }\end{array}$ & 25.2 & 26 & 10 & 2 & 40 & 1,09 \\
\hline
\end{tabular}

Nota: Elaboración propia en base a datos de la encuesta Latinobarómetro 2017.

A partir de los estadísticos descriptivos entregados en la tabla 3 , se puede visualizar que los datos e información son consistentes debido a la baja cantidad de datos perdidos por cada uno de los índices analizados. En ninguno de los índices la cantidad de datos perdidos supera el 10\%.

En relación a los estadísticos de la variable de confianza política, se puede indicar que nuestro país se caracterizaría por contar con una baja confianza, ya que el promedio es de 13 y se encuentra más cercano al valor máximo de este índice (16), el cual hace referencia a "Ninguna confianza". Lo anterior se corrobora considerando la mediana de dicho indicar, ya que un 50\% de los encuestados tendría una confianza política que fluctúa entre los 14 y 16 puntos. Así también la tendencia hacia la desconfianza se visualiza en el valor que posee la desviación típica.

En el caso del indicador de eficacia, las personas, con respecto al funcionamiento de la democracia y la economía, tenderían a encontrarse más cercanas a la insatisfacción, debido a que el promedio de este índice es de 5.7, lo cual es más cercano al valor máximo de 8, "Nada satisfecho". En este caso, los valores de la mediana, la cual es igual a 6, así como también el 
valor de la desviación típica (1.3), corroboran y reafirman que la tendencia es encontrarse insatisfechos con la eficacia del sistema político democrático y el funcionamiento de la economía en Chile.

En relación a la percepción del bienestar económico, la media alcanza un valor igual a 8.6. Dicho valor, a diferencia de los índices anteriores, se encontraría más alejado de aquel polo del índice que hace referencia a percepciones desfavorables por parte de los encuestados. No obstante, de igual forma, el valor del promedio es más cercano al valor máximo 15, el cual hace referencia a una percepción de que el país estaría mucho peor en términos económicos. En este caso, los datos también se agrupan más en torno al valor máximo, consignando que el valor de la mediana es de 9 y el de la desviación es de 2.

Por último, en el caso del índice de percepción de corrupción, al igual que en la medición de la percepción de bienestar económico, se evidencia una tendencia no tan acentuada de los encuestados con respecto a percibir mucha corrupción. Pese a ello, el promedio (25) es más cercano al valor máximo (40), el cual indica percepción de mucha corrupción en distintas instituciones políticas. En este caso, la desviación es bastante alta, de 10 puntos, debido a lo cual la percepción de los encuestados con respecto a lo consultado es más heterogénea.

\section{Resultados}

A partir de los resultados del modelo teórico, aplicado en una regresión lineal múltiple, podemos decir que la varianza explicada del modelo a partir del valor de R2 es de un $36 \%$. En tanto, el modelo posee una significación de 0.000 , lo cual posibilita su aprobación en términos estadísticos; por lo que es pertinente continuar con la interpretación con respecto a cada una de las variables consignadas en este proceso. En tanto, otro aspecto interesante tiene que ver con la ausencia de colinealidad de las variables, ya que en el caso de los regresores los va- 
lores del factor de inflación de la varianza (VIF) no superan el 1.7 (García, 2010).

A continuación, se presentan aquellos aspectos asociados a los coeficientes de las variables independientes con respecto a la dependiente. Ello posibilita visualizar el comportamiento de los índices entre sí y la capacidad de explicación de los mismos.

Tabla 4

Coeficientes Modelo de Regresión Múltiple

\begin{tabular}{|l|c|}
\cline { 2 - 2 } \multicolumn{1}{l|}{} & Confianza Política \\
\hline Constante & 5.73 \\
\hline Eficacia & \\
\hline (B) Beta & 0.50 \\
\hline Percepción de bienestar económico & 0.00 \\
\hline (B) & \\
\hline \multicolumn{1}{|c|}{ Sig-P. } & 0.31 \\
\hline Percepción de corrupción & 0.00 \\
\hline (B) & \\
\hline Sig-P. & 0.069 \\
\hline R & 0.00 \\
\hline R2 & 0.60 \\
\hline R2 Corregida & 0.36 \\
\hline Error Tip. Estimación & 0.36 \\
\hline VIF & 2.1768 \\
\hline
\end{tabular}

Nota: Elaboración propia en base a datos de la encuesta Latinobarómetro 2017.

En primer lugar, se puede señalar que en el modelo construido sí existe una relación lineal significativa entre la variable dependiente y aquellas independientes, ya que la significación alcanzada es de 0.000. En tanto, un aspecto importante es que el R2 alcanza un valor de 0.364 , así, también, el error de estimación es bajo.

En tanto, otro aspecto a destacar es que los residuales producidos en este modelo de regresión poseen un comportamiento normal; lo cual destaca una distribución normal de la variable 
dependiente con respecto a los valores predichos en el modelo de regresión lineal. Lo anterior se puede visualizar considerando el promedio del residual, el cual es de valor 0.

Tabla 5

Estadísticas residuos

\begin{tabular}{|l|l|l|l|l|l|}
\hline & Mínimo & Máximo & Media & Desv. & N \\
\hline Valor pronosticado & 8.2935 & 17.1671 & 13.0428 & 1.64373 & 989 \\
\hline Residuo & -8.55882 & 5.90643 & 0.0000 & 2.17349 & 989 \\
\hline $\begin{array}{l}\text { Desviación Valor } \\
\text { pronosticado }\end{array}$ & -2.889 & 2.509 & 0.000 & 1.000 & 989 \\
\hline Desviación residuo & -3.932 & 2.713 & 0.000 & 0.998 & 989 \\
\hline
\end{tabular}

Nota: Elaboración propia en base a datos de la encuesta Latinobarómetro 2017.

La normalidad de los residuos y la cantidad no significativa de casos atípicos en el modelo puede visualizarse mediante el siguiente histograma.

Gráfico 1

Histograma de variable dependiente, Confianza Politica

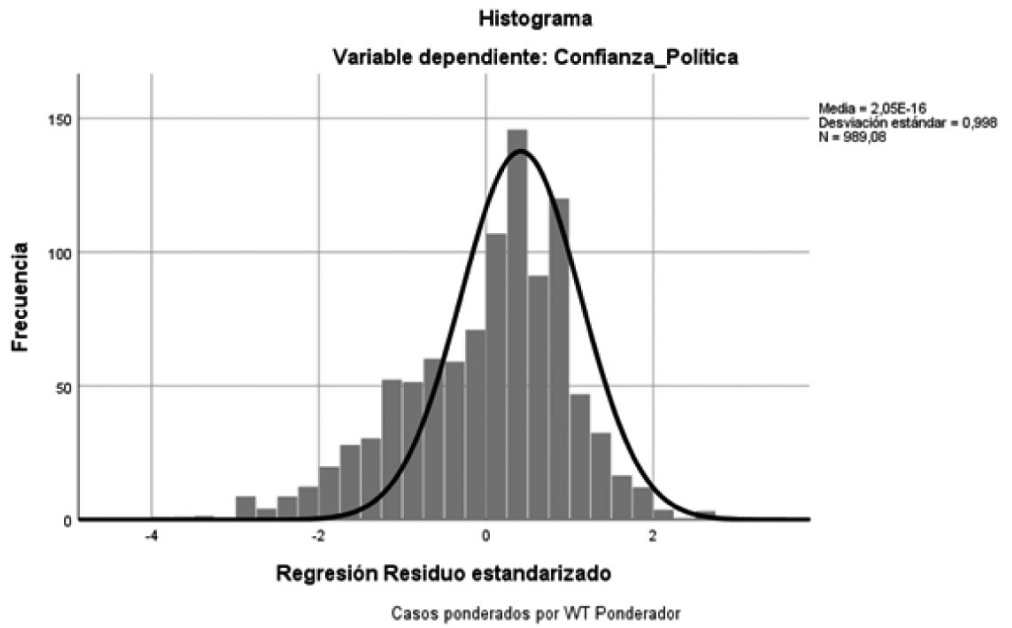

Nota: Elaboración propia en base a datos de la encuesta Latinobarómetro 2017. 
Respecto a los coeficientes del Modelo 1, el índice de eficacia es significativo en tanto su valor P es igual a 0.000. Respecto a la predicción entre la variable "eficacia" y "Confianza política", los resultados nos indican que, en promedio, el aumento en un punto en la evaluación de eficacia por parte de la muestra aumenta en un 0.505 promedio. A partir de estos resultados, se rechaza la hipótesis nula, en adelante H0, por lo que es posible afirmar que en la medida que los ciudadanos no están satisfechos con la actuación de las instituciones políticas respecto a temáticas democráticas y económicas, disminuye la confianza hacia ellos.

Respecto al predictor "percepción de bienestar económico", este es significativo en el modelo, ya que su valor P es igual a 0.000. Su relación con la variable dependiente de "confianza política" es proporcional. En tanto predice que, en promedio, el aumento de un punto el índice de "Percepción de bienestar económico" de la muestra considerada aumenta en un 0.310 promedio en con respecto a la variable dependiente de "Confianza política". A partir de estos resultados, es posible rechazar la H0. Por ello, es posible afirmar que aquellas personas que manifiestan una percepción negativa con respecto al bienestar económico tanto del país como personal-familiar, tienen una menor confianza en las entidades políticas trabajadas en este artículo.

El regresor de "Percepción de corrupción" es significativo en el modelo, ya que su valor P es igual a 0.000 . Su relación con la variable predicha es directamente proporcional, prediciendo, en promedio, que el aumento de un punto en la percepción de acceso a oportunidades aumenta en un 0.069 promedio la confianza en instituciones políticas. Por esta razón es posible rechazar la $\mathrm{H} 0$, afirmando que las personas que perciben un mayor grado de corrupción en las instituciones políticas poseen una menor confianza en las mismas.

Visualizando el comportamiento de los distintos predictores, con respecto a la variable independiente, se puede destacar que aquel que mayor influencia posee en el comportamiento de la 
confianza política es aquel indicador que hace referencia a la eficacia de las instituciones políticas: 0.505 . Considerando la pendiente del modelo, la cual posee un valor de 5.737, así también los betas de las distintas variables, se puede visualizar que el comportamiento de la recta de regresión es positiva y proporcional.

Considerando la fórmula de regresión lineal múltiple: $\mathrm{Y}=\mathrm{a}$ $+b 1 x+b 2 x+b 3 x$, sabiendo que $Y$ corresponde a la variable a explicar/predecir, es decir la "confianza política", mientras que " $a$ " refiere a la constante, en tanto que cada " $b$ " hace relación a los regresores, los cuales corresponden: "eficacia", "percepción de bienestar económico" y "percepción de corrupción". En tanto cada " $x$ " hace referencia a la variación de los puntajes en las variables independientes.

Por lo tanto, teniendo en cuenta el carácter proporcional positivo del comportamiento de la variable predicha, con respecto a sus regresores (García, 2000), se debe destacar que por el aumento de cada una de los betas de los predictores, aumenta el grado de desconfianza en instituciones políticas.

\section{Discusión y conclusiones}

Mediante la aplicación de un modelo de regresión lineal múltiple, el presente artículo se propuso explicar los niveles de confianza en las instituciones políticas en Chile por medio de los datos de la encuesta Latinobarómetro 2017, poniendo a prueba tres indicadores, definidos teóricamente, para explicar los niveles de confianza: la percepción de eficacia de las instituciones, bienestar económico y percepción de corrupción, dando cuenta así del potencial explicativo de este tipo de variables subjetivas en el caso de Chile, reconocidas por diferentes estudios de la confianza política actuales.

En conjunto, los resultados arrojados por este artículo confirman, en primera instancia, la pertinencia de estos indicadores, que aluden a un enfoque basado en la performance o desempeño 
institucional para explicar los niveles de confianza política en Chile. En segunda instancia, destaca la relevancia que tiene para los encuestados el modo de actuar de las instituciones y sus representantes, en tanto estos cumplen o no con los objetivos que se proponen, especialmente en lo que respecta a las variables económicas.

Sobre al primer indicador, la consideración de satisfacción con la democracia y funcionamiento de la economía, como factores relacionados con la eficacia de las instituciones políticas, comprueba la importancia del desempeño institucional en estas áreas para la confianza política, reafirmando su relevancia teórica para el caso chileno. Creemos que este resultado se debe a que los encuestados asocian la satisfacción con la democracia y la situación económica, principalmente, con el desempeño y decisiones del gobierno de turno. La provisión de servicios públicos y la promoción de derechos sociales por parte de los gobiernos puede ser un factor fundamental para que los ciudadanos se sientan satisfechos con la democracia y, con ello, fortalecer el vínculo de confianza con las instituciones (Corral, 2008).

Además, hay que considerar que estas medidas pueden fortalecer la percepción positiva del funcionamiento de la economía en la medida en que los ciudadanos identifiquen que estas iniciativas de las autoridades toman en cuenta sus necesidades y apuntan a aliviar su condición económica en materias tales como educación, salud, previsión social, etc. Claramente, esto depende de la efectividad de las medidas macroeconómicas de las autoridades, sobre todo en un contexto global en el que la desaceleración económica y la aplicación de políticas de austeridad puede tener importantes consecuencias para los países en desarrollo, sobre todo en la profundización de los niveles de desigualdad socioeconómica, que también se relaciona con los niveles de confianza política (Zmerli \& Castillo, 2015). Lo último resulta clave en el caso chileno, que presenta alto niveles de desigualdad socioeconómica que en los últimos años ha alimentado el surgimiento de movimientos sociales que articulan sus objetivos en pos de 
combatirla a través de la articulación de demandas por recuperación de derechos sociales.

Por su parte, la percepción de bienestar económico, confirma su importancia como variable explicativa de los niveles de confianza política ya señalados en otros estudios (Morales, 2008; Norris \& Inglehart, 2019). En este caso, no solo consideramos la evaluación de la situación económica personal actual sino que también la evaluación retrospectiva y proyectiva de la situación económica del país. Consideramos que ello entrega una mayor dimensión al indicador en términos temporales, ya que toda percepción sobre una situación presente contempla una comparación con su pasado para definir los criterios sobre si es mejor o peor y, a su vez, considerar las expectativas futuras da cuenta del grado de optimismo o pesimismo respecto a sus condiciones económicas presentes. La importancia de esta variable a nivel individual, para la confianza institucional en Chile, puede relacionarse con el ingreso económico personal y familiar y cómo es contrastado con la percepción que se tenga del ingreso de otros sectores sociales. Su efecto puede resultar negativo para la confianza política en Chile, dado los niveles de inequidad en la distribución del ingreso, lo que pone en tela de juicio la acción de las autoridades políticas en la búsqueda de soluciones al problema.

Finalmente, el indicador de percepción de corrupción, que teóricamente presentaba las mayores dudas respecto a su validez como predictor de la confianza política, demuestra su capacidad explicativa, tal como lo han hecho ya estudios recientes en esta materia (Jiménez, 2016; Simón, 2019). El efecto de corrupción sobre la confianza política en el caso chileno puede relacionarse con la aparición en la prensa de múltiples casos en que representantes políticos y autoridades de instituciones se han visto involucrados en conflictos de interés y corrupción (Cantillana \& Castañeda, 2015; Sola-Morales \& Rivera, 2017), particularmente por desvío de fondos públicos y financiamiento ilícito por parte de grupos empresariales y privados, que han dejado al descubierto el poder de influencia de estos sectores económicos por 
la obtención de prebendas políticas, así como también casos de corrupción económica y financiera, tales como fraudes tributarios y las colusiones que dejan en entredicho los mecanismos de autorregulación del sistema político que son vulnerados. Consideramos que estas situaciones han impactado negativamente en la opinión pública general respecto a la política, no solo aumentando la brecha ya existente con la ciudadanía, sino que, alimentando el surgimiento de pasiones políticas negativas como la desconfianza, la rabia y la indignación (Arias-Maldonado, 2016). En este sentido, es necesario que las autoridades e instituciones apunten a fortalecer aún más los mecanismos de regulación y transparencia activa que eviten el cruce de intereses públicos y privados que puedan poner el riesgo la credibilidad de las instituciones políticas y, con ello, la legitimidad democrática.

Con respecto al diseño de este estudio, cabe mencionar algunas dificultades y limitaciones que otras investigaciones pueden subsanar. Por un lado, dadas las limitaciones por las preguntas presentes en la encuesta, fue imposible construir un índice de eficacia y eficiencia en las instituciones políticas que aludiera efectivamente en la evaluación de sus funciones y acciones y de la capacidad para cumplir sus propósitos, los que hubieran sido más precisos para este constructo. Sin embargo, los indicadores escogidos resultan también pertinentes, no solo porque estadísticamente así se ha probado sino porque la satisfacción con la democracia y con el funcionamiento de la economía también son aspectos que van de la mano con la gestión de las instituciones y sus autoridades. Por otro lado, la ausencia de un indicador en el modelo sobre la perspectiva cultural de la confianza política puede restar capacidad explicativa en tanto no explora esta variable. La decisión de no incluir esta variable, y otras en el modelo, se debió a la poca pertinencia de las preguntas relacionadas a esta dimensión en la encuesta Latinobarómetro 2017.

Como conclusión y reflexión final, consideramos necesario para el caso de Chile una mayor preocupación por fortalecer el lazo social básico representando en la confianza en las institucio- 
nes políticas, sobre todo en medio del creciente malestar social presente en la población, reflejado en el aumento de la conflictividad social y de movimientos sociales (PNUD, 2015) en esta década. Si bien resulta dificultoso pensar una solución efectiva a la desafección de los ciudadanos hacia la política institucional que ha encontrado reverso en la participación en formas no convencionales, esto habla de individuos con capacidades críticas mayores ante el régimen democrático y sus actores institucionales (Norris, 2011). Un camino posible para recuperar progresivamente la confianza en las instituciones es establecer políticas que atiendan aspectos estructurales en lo que respecta al funcionamiento del sistema democrático y sus mecanismos de inclusión de la ciudadanía en toma de decisiones y de regulación y probidad para sus instituciones, así como también en el modelo de desarrollo económico vigente que, a la fecha, sigue acrecentando la brecha de desigualdad de ingresos y riqueza. Pese a que Chile presenta una economía estable, parece ser que la población no se encuentra satisfecha y no se refleja el progreso macroeconómico del país en su bienestar económico personal, lo que sigue haciendo crecer el descrédito de las autoridades políticas y sus instituciones en tanto no toman acciones profundas para atender problemáticas de desigualdad. En consecuencia, urge que las autoridades y representantes de las instituciones políticas consideren prioritario fortalecer su credibilidad y que sus acciones se orienten a atender las demandas y problemas de la sociedad civil a fin de recuperar progresivamente la confianza política que resulta fundamental para la convivencia y estabilidad democrática.

\section{Referencias}

Almond, G. \& Verba, S. (1989). The civic culture: Political attitudes and democracy in five nations. Londres: Sage.

Anderson, C. \& Tverdova, Y. (2003). Corruption, political allegiances, and attitudes toward government in contemporary democracies. American Journal of Political Science, 47(1), 91-109. 
Aninat, I., \& González, R. (2016). ¿Existe una crisis institucional en el Chile actual? Puntos de referencia, (440), 1-13.

Arias-Maldonado, M. (2016): La democracia sentimental. Política y emociones en el siglo XXI. Barcelona: Página Indómita.

Cantillana, C. \& Castañeda, J. (2015). Escándalos políticos y su efecto sobre el desempeño electoral de los candidatos a la Cámara de Diputados en las legislativas chilenas de 2013. Trabajo preparado para su presentación en el VIII Congreso Latinoamericano de Ciencia Política, organizado por la Asociación Latinoamericana de Ciencia Política (ALACIP). Pontificia Universidad Católica del Perú, Lima, 22 al 24 de julio de 2015.

Centro de Estudios de Conflicto y Cohesión Social (COES). (2015). Encuesta COES. Tema 1: ¿Crisis en la confianza politica? Disponible en: http://www.coes.cl/wp-content/uploads/2017/01/ modulo1.pdf

Centro de Estudios Públicos (CEP). (2017). Estudio Nacional de Opinión Pública $\mathrm{N}^{\circ}$ 79. Disponible en: https://www.cepchile.cl/ cep/site/artic/20170601/asocfile/20170601155007/encuestacep_abr_may2017.pdf

Chang, E. \& Chu, Y. (2006). Corruption and trust: Exceptionalism in Asian democracies? Journal of Politics, 68(2), 259-271.

Corbetta, P. (2007). Metodología y técnicas de investigación social. España: Mc-GrawHill.

Corral, M. (2008). (Des)confianza en los partidos políticos en América Latina. Revista de Ciencia Política (Santiago), 28(2), 195-202.

Cossarini, P. \& Vallespín, F. (2019). Populism and passions. Democratic legitimacy after austerity. Nueva York: Routledge.

Crouch, C. (2004). Post-Democracy. Oxford: Polity.

Dalton, R. (2004). Democratic challenges, democratic choices: The erosion of political support in advanced industrial democracies. Oxford: Oxford University Press.

del Tronco, J. (2012). La causa de la desconfianza política en México. Perfiles Latinoamericanos, (40), 227-251.

Della Porta, D. (2000). Social capital, beliefs in government, and political corruption. En S. J. Pharr \& R. D. Putnam (Eds.), Disaffected democracies: What's troubling the trilateral countries? (pp. 202227). Princeton: Princeton University Press. 
Durand, V. (2006). Confianza y eficacia ciudadana en una sociedad con alta desigualdad. Opinião Pública, 12(2), 277-296.

Easton, D. (1975). A re-assessment of the concept of political support. British Journal of Political Science, 25(4), 485-514.

Economist Intelligence Unit. (2018). Democracy Index 2018: Me too? Political participation, protest and democracy. Londres: Economist Intelligence Unit. Disponible en: https://www.eiu.com/ topic/democracy-index

Elinas, A. \& Lamprianou, I. (2014). Political Trust In Extremis. Comparative Politics, 46(2), 231-250.

Freedom House. (2015). Freedom in the World. Disponible en: https:/ / freedomhouse.org/report/freedom-world/freedomworld-2015

García, M. (2000). Socioestadística. Introducción a la estadística en sociología. Madrid: Alianza Editorial

Hakhverdian, A. \& Mayne, Q. (2012). Institutional trust, education, and corruption: A micro-macro interactive approach. Journal of Politics, 74(3), 739-750.

Hardin, R. (2010). Confianza y confiabilidad. México D. F.: Fondo de Cultura Económica.

Jiménez, J. F. (2013). Crisis económica, confianza institucional y liderazgos políticos en España. Barataria. Revista Castellano-Manchega de Ciencias Sociales, (15), 125-141.

Jiménez, J. F. (2016). La corrupción y sus dimensiones. En F. Llera-Ramo (Ed.), Desafección política y regeneración democrática en la España actual: Diagnósticos y propuestas, (pp. 247-276). Madrid: Centro de Estudios Políticos y Constitucionales.

Joignant, A., Morales, M. \& Fuentes, F. (2017). Malaise in representation in Latin American countries: Chile, Argentina, and Uruguay. Nueva York: Palgrave.

Jörke, D. (2008). Post-democracia en Europa y América Latina. Revista de Sociología, (22), 141-156.

Kroknes, V., Jakobsen, T. \& Grønning, L. (2015). Economic performance and political trust: The impact of the financial crisis on European citizens. European Societies, 17(5), 700-723.

Lechner, N. (2016). Los patios interiores de la democracia. En N. Lechner (Ed.), Obras III. Democracia y utopía: la tensión permanente. México D. F.: Fondo de Cultura Económica. 
Levi, M. \& Stoker, L. (2000). Political trust and trustworthiness. Annual Review of Political Science, 3(1), 475-507.

Lipset, S. (1977). El hombre político. Las bases sociales de la política. Buenos Aires: Editorial Universitaria.

Listhaug, O. \& Ringdal, K. (2008). Trust in political institutions. En H. Ervasti, T. Fridberg, M. Hjerm \& K. Ringdal (Eds.), Nordic social attitudes in a European perspective (pp. 131-151). Cheltenham: Edward Elgar.

López, M. \& Gutiérrez, L. (2018). Cómo realizar e interpretar un análisis factorial exploratorio utilizando SPSS. Revista d'Innovació $i$ Recerca en Educació, 12(2), 1-14.

Marien, S. (2011). Measuring political trust across time and space. En M. Hooghe \& S. Zmerli (Eds.), Political trust. Why context matters, (pp. 13-46). Colchester: ECPR Press.

Megías, A. (2019). Desafección política: ¿estructura o coyuntura? Un estudio profundo en el contexto actual de crisis. (Tesis Doctoral). Universidad de Murcia, España.

Miller, A. \& Listhaug, O. (1990) Political parties and confidence in government: A comparison of Norway, Sweden, and the United States. British Journal of Political Science, 20(3), 357-386.

Mishler, W. \& Rose, R. (2001). What are the origins of political trust? Testing institutional and cultural theories in post-communist societies. Comparative Political Studies, 34(1), 30-62.

Mishler, W., \& Rose, R. (2005). What Are the Political Consequences of Trust?: A Test of Cultural and Institutional Theories in Russia. Comparative Political Studies, 38(9), 1050-1078.

Montero, J., Zmerli, S. \& Newton, K. (2008). Confianza social, confianza política y satisfacción. Revista Española de Investigaciones Sociológicas, (122), 11-54.

Morales, M. (2008). Evaluando la confianza institucional en Chile. Una mirada desde los resultados LAPOP. Revista de Ciencia Política, 28(2), 161-186.

Newton, K. \& Norris, P. (2000). Confidence in public institutions: Faith, culture, or performance? En S. Pharr \& R. Putnam (Eds.), Disaffected democracies: What's troubling the trilateral countries?, (pp. 52-73). Princeton: Princeton University Press.

Norris, P. \& Inglehart, R. (2018). Cultural backlash: Trump, brexit and authoritarian populism. Cambridge: Cambridge University Press. 
Norris, P. (2011). Democratic Deficit. Critical Citizens Revisited. Cambridge: Cambridge University Press.

Norris, S., \& Klesner, J. (2010). Corruption and trust: Theoretical considerations and evidence from Mexico. Comparative Political Studies, 43(10), 1258-1285.

Offe, C. (2014). Posdemocracia en la era de los mercados financieros globales. Pasajes: Revista de Pensamiento Contemporáneo, (43), 154-162.

Park, C. (2017). Political trust in the Asia-Pacific region. En S. Zmerli \& T. W. G. van der Meer, (Eds.), Handbook on political trust, (pp. 488-508). Cheltenham: Edward Elgar.

PNUD (Programa de las Naciones Unidas para el Desarrollo). (2015). Desarrollo Humano en Chile: Los tiempos de la politización. Santiago de Chile: PNUD.

Przeworski, A., Álvarez, M., Cheibub, J. \& Limongi, F. (1996). What makes democracies endure? Journal of Democracy, 7(1), 39-55.

Putnam, R. (2011). Para que la democracia funcione: Las tradiciones cívicas en la Italia moderna. Madrid: Centro de Investigaciones Sociológicas.

Rose-Ackerman, S. (1999). Corruption and government: Causes, consequences, and reform. Cambridge: Cambridge University Press.

Runciman, D. (2019). Así terminan las democracias. Barcelona: Paidós.

Segovia, C. (2006). Percepciones ciudadanas y calidad de la democracia en Chile. En C. Fuentes \& A. Villar (Eds.), Desafíos democráticos (pp. 87-132). Santiago de Chile: FLACSO-Chile, LOM Ediciones.

Siavelis, P. (2016). Crisis of representation in Chile? The institutional connection. Journal of Politics in Latin America, 8(6), 61-93.

Simón, P. (2019). De la crisis económica a la crisis política: Cambio en Europa y España. Información Comercial Española, ICE: Revista de economía, (906), 125-138.

Sola-Morales, S. \& Rivera, R. (2016). El tratamiento periodístico sobre la corrupción política. Análisis comparado del caso SQM en Chile y del caso Bárcenas en España. Estudios sobre el mensaje periodístico, 23(1), 647-662.

Stoyan, A., Niedzwiecki, S., Morgan, J., Hartlyn, J. \& Espinal, R. (2016). Trust in government institutions: The effects of performan- 
ce and participation in the Dominican Republic and Haiti. International Political Science Review, 37(1), 18-35.

Torcal, M. (2014). The decline of political trust in Spain and Portugal: Economic performance or political responsiveness? American Behavioral Scientist, 58(12), 1542-1567.

van der Meer, T. \& Deeker, P. (2011). Trustworthy states, trusting citizens? En S. Zmerli \& M. Hooghe (Eds), Political trust: why context matters, (pp. 95-116). Colchester: ECPR Press.

van der Meer, T. \& Hakhverdian, A. (2017) Political trust as the evaluation of process and performance: A cross-national study of 42 European countries. Political Studies, 65(1), 81-102

van der Meer, T. (2010). In what we trust? A multi-level study into trust in parliament as an evaluation of state characteristics. International Review of Administrative Sciences, 76(3), 517-536.

van der Meer, T. (2018). Economic performance and political trust. En E. M. Uslaner (Ed.), The Oxford handbook of social and political trust, (pp. 599-616). Nueva York: Oxford University Press.

Vilhelmsdóttir, S. \& Helgi G. (2018) Political trust in Iceland: Performance or politics? Icelandic Review of Politics and Administration, 14(1), 211-234.

Wroe, A., Allen, N. \& Birch, S. (2012). The role of political trust in conditioning perceptions of corruption. European Political Science Review, 5(2), 175-195.

Zmerli, S. \& Castillo, J. (2015). Inequality and political trust. How objective and subjective inequality affects Latin American democracies. Social Science Research, (52), 179-192. 